\title{
IS THE OUTBURST OF U SCORPII
}

AN ACCRETION EVENT?

$$
\text { R.F. Webbink }
$$

Department of Astronomy

University of Illinols

A summary of the peculiarities of the spectral evolution of the recurrent nova $\mathrm{T}$ Cr $B$ are given, and interpreted in terms of an episodic accretion model. On the basis of the marked similarity of U Sco with this nova, it is suggested that the observed maximum will be followed by a secondary maximum, reaching $V=14-15$. Such a secondary maximum should be very different for an accretion event (showing a strong continuum) and a thermonuclear outburst (showing a nebular spectrum). If observations of a star at 14th magnitude at the position of $U$ Sco in February 1969 were indeed of a secondary maximum of this nova, the principal maximum probably precedes the secondary by less than 65 days. 